\title{
Subtle Aortic Dissection in a Patient with Severe Aortic Regurgitation and Undiagnosed Bicuspid Aortic Valve: Case Report with a Literature Review
}

\author{
Davorin Sef ${ }^{1}$, Stewart Brown ${ }^{2}$, Omar Jarral ${ }^{1}$, Azhar Hussain ${ }^{1}$, Elizabeth Haslam² ${ }^{2}$ Cha \\ Rajakaruna $^{3}$, and Christopher McAloon ${ }^{2}$ \\ ${ }^{1}$ St Bartholomew's Hospital \\ ${ }^{2}$ Gloucestershire Royal Hospital \\ ${ }^{3}$ Bristol Royal Infirmary
}

April 12, 2021

\begin{abstract}
A subtle aortic dissection can be challenging to detect despite the availability of multiple diagnostic modalities. Whilst rare, the inability to detect this variant of aortic dissection can lead to a dismal prognosis. We present an extremely rare case of a subtle aortic dissection with supraannular aortic root intimal tear and acute severe aortic regurgitation in a patient with a bicuspid aortic valve. Initial concerns were either aortic dissection or infective endocarditis. Despite advanced multimodality preoperative imaging, diagnosis was made intraoperatively and a Bentall procedure with a mechanical aortic valve was performed. As current data is limited, a literature review concerning subtle aortic dissection is provided.
\end{abstract}

\section{INTRODUCTION}

In patients with a classic intimal flap tear causing an aortic dissection, diagnosis by available imaging tests is accurate with extremely high sensitivity and specificity [1-3]. The entity presented here is an extremely rare variant of aortic dissection that was characterized by a supraannular intimal tear without further progression and separation of the medial layers or an intimal flap.

\section{CASE PRESENTATION}

A 41-year-old Africo-Caribbean female with a body mass index of $29 \mathrm{~kg} / \mathrm{m}^{2}$ presented to the emergency department with acute pulmonary oedema and increasing dyspnea on exertion with New York Heart Association (NYHA) class 3 symptoms. The patient initially experienced an episode of sudden onset tight central chest pain while walking, followed by four weeks of fatigue and malaise and two weeks of exertional breathlessness, orthopnea and a productive nocturnal cough. She had been treated empirically with antibiotics for a chest infection, but her symptoms deteriorated prompting presentation to her local hospital. The patient had essential hypertension with subsequent chronic kidney disease and pre-eclampsia during her pregnancy. She had no previous history of congenital disease, trauma, or positive family history for aorthopathy.

Her vital parameters were stable and she was apyrexial. She had bilateral basal lungs crepitations. The patient's electrocardiogram was normal. Her leukocyte count was $8.4 \times 109 / \mathrm{L}$, D-Dimer $2522 \mathrm{ng} / \mathrm{mL}$, troponin $13.2 \mathrm{ng} / \mathrm{L}$, Covid-19 swab test and blood cultures were negative. Chest radiograph showed pulmonary oedema with bilateral pleural effusions. Transthoracic echocardiography (TTE) demonstrated a normal size left ventricle with hyperdynamic systolic function and severe aortic regurgitation (AR) with a central jet. There was an appearance of a trileaflet aortic valve (AV) with failure of coaptation along the right 
coronary/noncoronary cusp (RCC/NCC) closure line (Figure 1). The coaptation defect was associated with a linear echogenic structure attached to the RCC, running along the closure line. This linear and thinned structure was mobile (Figure 1). No prolapse of the AV leaflets was demonstrated and the aortic root was mildly dilated with no signs of dissection flap. Transesophageal echocardiography was attempted, although the patient refused to have it. A prospectively gated computed tomography (CT) aortogram showed pulmonary oedema with an asymmetrical aortic root dilatation with increased RCC-LCC diameter (maximum $45 \mathrm{~mm}$ ) and no features of aortic dissection or pulmonary embolus (Figure 2). Given the history of acute severe AR and treatment for chest infection, the initial concerns were for infective or non-infective endocarditis (Libman-Sacks endocarditis).

The patient was transferred to a tertiary cardiothoracic surgical center for urgent surgical treatment in view of acute AR. After median sternotomy, cardiopulmonary bypass (CPB) was initiated with cannulation of the distal ascending aorta and right atrium with mild hypothermia $\left(34^{\circ} \mathrm{C}\right)$. Intermittent antegrade cold blood cardioplegia were delivered. After atriotomy, a supraannular aortic intimal tear starting from the RCC/NCC commissure was found, which did not extend into the ascending aorta (Figure 3). The AV on direct inspection was bicuspid with an RCC/NCC raphe involved in the intimal tear. Aortic root and ascending aorta were found dilated with a diameter up to 45 and $40 \mathrm{~mm}$, respectively. Severe AR not amenable to repair was found. Bentall procedure was performed using the $23 / 26 \mathrm{~mm}$ Carbomedics Carbo Seal Valsalva composite graft (Sulzer Carbomedics Inc, Austin, TX, USA). The patient was weaned from CPB with no inotropic support. CPB and clamp time were 109 and 86 minutes, respectively. Patient was anticoagulated with warfarin and had uneventful postoperative recovery. Valve tissue samples and blood cultures were negative. Histopathological examination revealed focal mucinous degeneration in the aortic root media with no connective tissues disorder. She was discharged on the $7^{\text {th }}$ postoperative day with a satisfactory TTE and remained well during a 6-month follow-up. Informed consent and patient's permission were obtained to report this case.

\section{Discussion}

To our knowledge, this is the first publication of subtle aortic dissection with a supraannular aortic root intimal tear in a patient with a dilated aortic root and undiagnosed bicuspid aortic valve that necessitated treatment with a Bentall procedure. This young lady developed acute severe AR and pulmonary oedema due to subtle aortic dissection. However, the patient was initially misdiagnosed and treated for a lower respiratory tract infection. Prior to presenting to her local hospital, she suffered an episode of tight central chest pain and this is likely to be the event when she potentially developed subtle aortic dissection but, despite two CT aortograms, no dissection was detected.

Svensson et al. reported that 9 out of 181 patients who underwent ascending or aortic arch repair had an undiagnosed aortic intimal tear without hematoma [4]. In all patients, this specific variant of aortic dissection characterized with a stellate or linear intimal tear was not proven by multiple noninvasive imaging techniques and diagnosis was made intraoperatively [4]. They also found that a subtle eccentric bulge or bubble was detected on aortography in 6 out of 9 patients which suggested that aortography should be considered if the more subtle form of aortic dissection is suspected based on clinical presentation. Furthermore, the authors reported that if multimodality imaging tests fail to detect aortic dissection and an ascending aortic aneurysm $>4.5 \mathrm{~cm}$ in diameter is measured, particularly if the patient has Marfan syndrome, Ehlers-Danlos syndrome, or a pericardial effusion, then an invasive imaging test such as aortography should be performed [4]. However, our patient had the suspicion of non-infective or infective endocarditis due to the suggestion of a small echogenic mass with the acute severe AR on TTE and had a bicuspid AV with a supraannular intimal tear found at the time of surgery. Wang et al. have described a patient with an annular intimal tear of the aortic root that was misdiagnosed as AR [5]. Similarly, preoperative imaging was inadequate for correct diagnosis which was confirmed intraoperatively. However, their patient underwent successful repair of the aortic root tear with Teflon felt, as the aortic root was not dilated. On the other hand, Shingal et al. have reported a patient that developed aortic intimal tear with severe AR secondary to subadventitial rupture of ascending aorta and no signs of dissection flap who underwent a Bentall procedure, but in their case it was 
a result of blunt trauma [6]. Manuchehry et al. have reported a case of fatal cardiac tamponade caused by a limited aortic dissection variant that was not detected by multiple imaging modalities [7]. However, their patient had the diagnosis confirmed during an autopsy.

\section{Conclusions}

Subtle aortic dissection is rare and are invariably missed despite the use of advanced imaging modalities. Our patient had suspected aortic valve endocarditis (infective or non-infective) combined with a dilated aortic root. Diagnosis was only made intra-operatively through inspection and successfully treated with a Bentall procedure. Our case along with the review of current literature emphasizes that current imaging techniques may be inadequate for diagnosis of this rare variant of aortic dissection.

Author contributions: DS: Conceptualization; Investigation; Methodology; Writing - Original Draft; Writing-Review \& Editing.SB,OJ,AH,EH,CR,CM: Conceptualization, Investigation; Methodology; Writing-Review \& Editing.

\section{Data Availability Statement}

Data available on request due to privacy/ethical restrictions.

Figure Legends

Figure 1: Transthoracic echocardiogram. (a) Parasternal long axis view of AV demonstrating severe AR. (b) The parasternal short axis view with color Doppler demonstrating the appearance of a trileaflet AV with failure of coaptation along the $\mathrm{RCC} / \mathrm{NCC}$ closure line.

Figure 2: Gated CT coronary angiography.

(a) CT axial reconstruction demonstrating apparent trileaflet morphology of the AV.

(b) Axial reconstruction perpendicular to centerline through the aortic root, demonstrating root dilatation and asymmetrical prominence of the left coronary sinus.

(c) Longitudinal reconstruction through the aortic root confirming asymmetrical root dilatation with increased RCC-LCC diameter (maximum 45mm).

Figure 3: Intraoperative view: (a) Aortic root exposure with a supraannular aortic intimal tear above the RCC/NCC commissure. (b) Excised supraannular aortic root intimal defect.

\section{References:}

1. Hiratzka LF, Bakris GL, Beckman JA, et al. 2010 ACCF/AHA/AATS/ACR/ASA/SCA/SCAI/SIR/STS/SVM Guidelines for the diagnosis and management of patients with thoracic aortic disease. A Report of the American College of Cardiology Foundation/American Heart Association Task Force on Practice Guidelines, American Association for Thoracic Surgery, American College of Radiology,American Stroke Association, Society of Cardiovascular Anesthesiologists, Society for Cardiovascular Angiography and Interventions, Society of Interventional Radiology, Society of Thoracic Surgeons, and Society for Vascular Medicine. J Am Coll Cardiol 2010;55(14):27-129.

2. Gologorsky E, Karras R, Gologorsky A, Ricci M, Salerno TA. Transesophageal echocardiography after contrast-enhanced CT angiography in the diagnosis of type A aortic dissection. J Card Surg 2011;26(5):495500.

3. Masri A, Bianco V, Kilic A, Gleason TG, Sultan I. Failure of CT angiogram to detect an ascending aortic dissection. J Card Surg 2018;33(4):194-195.

4. Svensson LG, Labib SB, Eisenhauer AC, Butterly JR. Intimal tear without hematoma: an important variant of aortic dissection that can elude current imaging techniques. Circulation 1999;99(10):1331-6. 
5. Wang $\mathrm{C}, \mathrm{Xiu} \mathrm{Z}, \mathrm{Gu} \mathrm{T}$. Annular intimal tear of the aortic root misdiagnosed as aortic regurgitation. J Thorac Cardiovasc Surg 2011;142(3):706-7.

6. Shingal P, Kejriwal N. Ascending aortic tear with severe aortic regurgitation following rugby injury. Heart, Lung and Circulation 2009;18:133-162

7. Manuchehry A, Fontana GP, Gurudevan S, Marchevsky AM, Siegel RJ. Missed diagnosis of limited ascending aortic dissection by multiple imaging modalities leading to fatal cardiac tamponade and aortic rupture. Echocardiography 2011;28(9):E187-90.

\section{Hosted file}

Figure 1 TTE.pdf available at https://authorea.com/users/362143/articles/517723-subtleaortic-dissection-in-a-patient-with-severe-aortic-regurgitation-and-undiagnosedbicuspid-aortic-valve-case-report-with-a-literature-review

\section{Hosted file}

Figure 2 CT.pdf available at https://authorea.com/users/362143/articles/517723-subtle-aorticdissection-in-a-patient-with-severe-aortic-regurgitation-and-undiagnosed-bicuspidaortic-valve-case-report-with-a-literature-review 


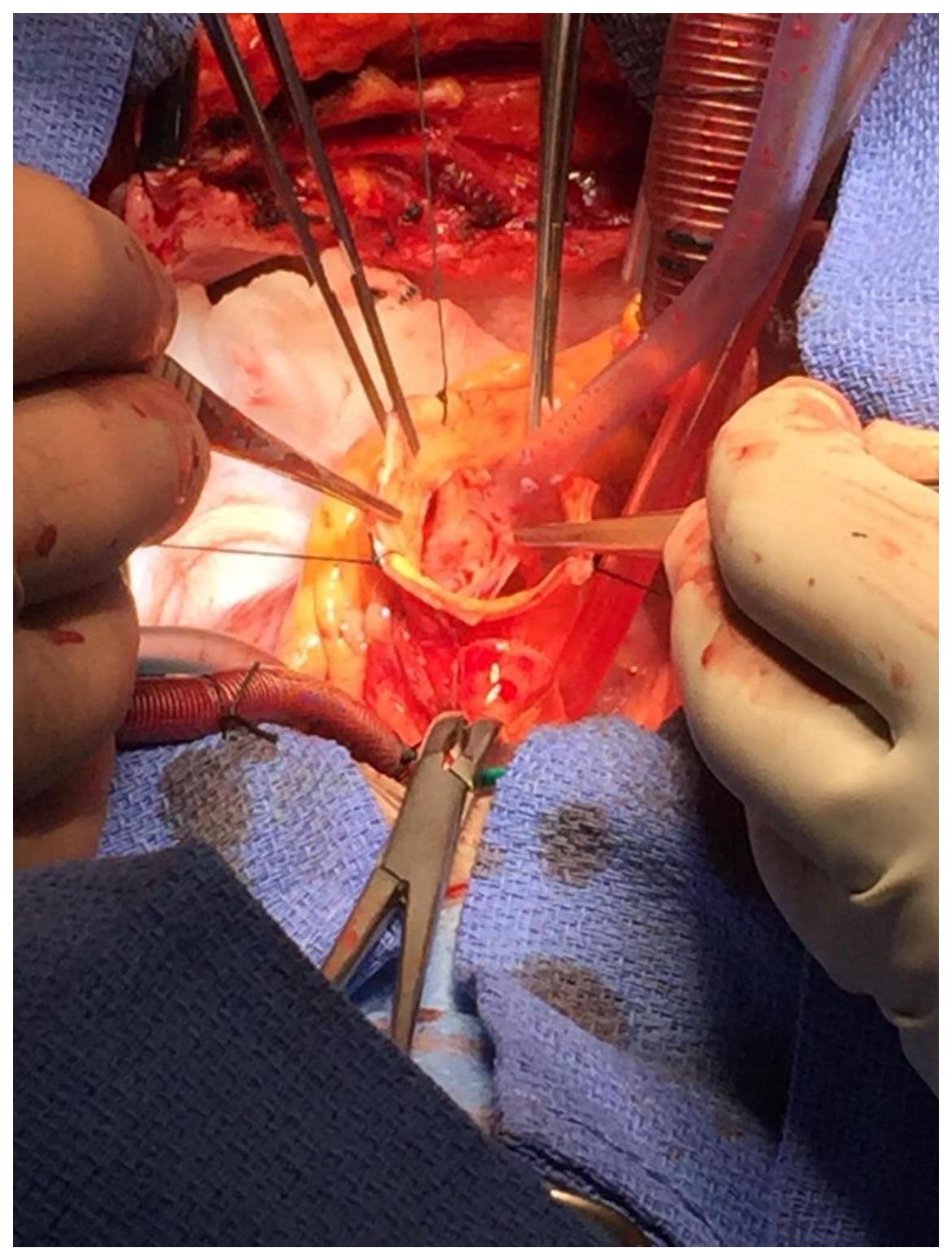




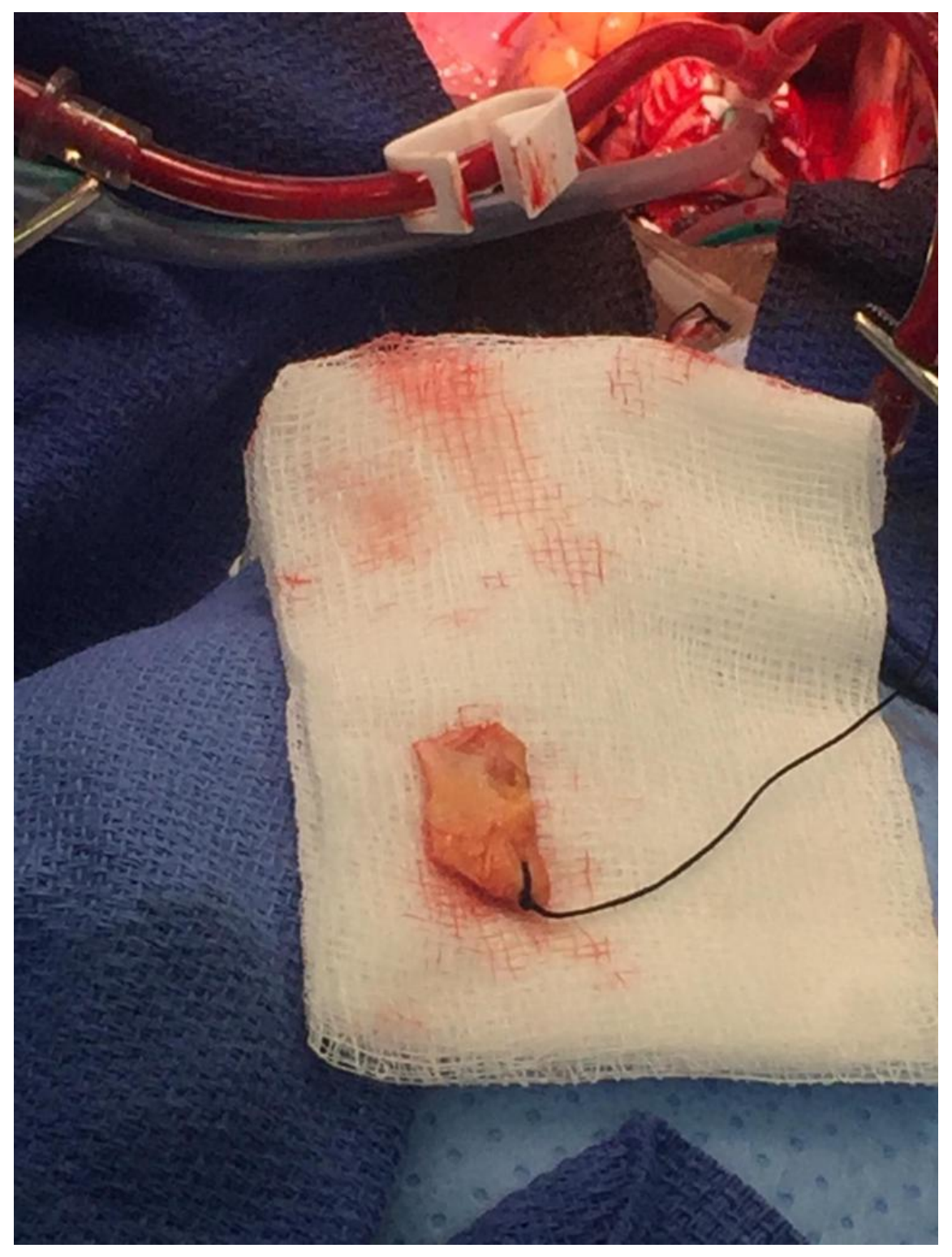

